\title{
A locus-driven mechanism for rapid and automated atlas- assisted analysis of functional images by using the Brain Atlas for Functional Imaging
}

\author{
Wieslaw L. Nowinski, D.Sc., Ph.D., and Arumugam Thirunavuukarasuu, B.Sc. \\ Biomedical Imaging Laboratory, Institute for Infocomm Research, Singapore
}

\begin{abstract}
Object. Functional imaging is an established neurosurgical modality for studying the brain in health and disease. Identifying numerous activation loci on many functional images and reading their underlying cortical and subcortical anatomy, coordinates, and anatomical and functional values is a tedious, time-consuming, and error-prone task. In this study the authors propose a novel approach to this problem by using an electronic brain atlas in conjunction with a locus-driven mechanism.

Methods. The Brain Atlas for Functional Imaging containing an enhanced and extended electronic version of the Talairach-Tournoux brain atlas was used for analysis. It enables loading of anatomical and functional data, correlation of these data, identification of activation loci, and their labeling with Brodmann areas, gyri, and subcortical structures by means of the atlas. The Talairach proportional grid system transformation is used to register the anatomical and functional data with the atlas. The availability of numerous tools supports this process.

A locus-driven mechanism for analysis of activation loci is implemented. Locus placement within the activation region is supported by thresholding, and its location can be further edited in three dimensions on any orthogonal plane. Once all loci are identified and edited, their labels, coordinates, and anatomical/functional values are read automatically and saved in an external file. This mechanism enables the analysis to be performed in an automated, rapid, explicit, three-dimensionally consistent, and user-friendly way.

Conclusions. The electronic brain atlas with locus-driven mechanism is a useful tool for localization analysis of functional images.
\end{abstract}

\section{KEY WORDS • brain atlas • Talairach-Tournoux brain atlas • localization analysis • activation locus • functional imaging}

During the last decade functional imaging has evolved from a novel research tool to an established neurosurgical modality for studying the healthy brain and for evaluating a variety of neurological diseases. The human brain varies highly among individuals, and comparison of individual brain requires one to do the following: 1 ) establish reference brain into which the results are normalized; 2) define a transformation that warps a given brain into the reference brain; and 3) establish a brain coordinate system. The brain atlas, brain normalization transformation, and stereotactic coordinate system introduced in the Co-Planar Stereotactic Atlas of the Human Brain ${ }^{24}$ are familiar and their application is widespread. This atlas, based on the anteroposterior commissural system, contains gross anatomical features on axial, coronal, and sagittal sections. Developed initially for stereotactic and functional neurosurgery, it is also widely used in human brain mapping. ${ }^{10}$ The Talairach system is the most prevalent coordinate system in human brain mapping, and the number of refer-

Abbreviations used in this paper: $\mathrm{BAFI}=$ Brain Atlas for Functional Imaging; fMR = functional magnetic resonance; $3 \mathrm{D}=$ three-dimensional. ences to the Talairach-Tournoux atlas continues to grow exponentially. ${ }^{5}$

Numerous software packages exist for the generation, processing, and analysis of functional images. ${ }^{2,3,6-8,22,23} \mathrm{~A}$ more comprehensive list of software packages was previously published, ${ }^{21}$ and a comparative analysis of fMR imaging statistical software packages has been published. ${ }^{9}$ None of the existing packages, however, contains a brain atlas or provides suitable tools for atlas-assisted localization analysis.

We have developed the BAFI to perform a rapid and user-friendly atlas-assisted analysis of functional images. $^{21}$ The BAFI contains a fully color-coded, labeled, extended, and enhanced Talairach-Tournoux brain atlas in axial, coronal, and sagittal orientations, as well as Brodmann areas ${ }^{1}$ and gyri in axial orientation. It is able to load one anatomical and a corresponding functional data set (in TIFF and AVW-Analyze file formats), interactively place the Talairach landmarks in 3D space, and automatically warp the data to the atlas by using the Talairach proportional grid system transformation. The accuracy of warping can be subsequently fine tuned interactively. The anatomical image, functional image, and atlas plate fused 
together are displayed as a single image with atlas-anatomy and anatomy-function blending controlled independently. The data warped to the atlas can be labeled interactively with the names of subcortical structures, gyri, and Brodmann areas. In addition, triplanar display and navigation, in which one plane from the data and the other two from the atlas, facilitates understanding of 3D relationships. The activation loci can be marked interactively, and this process is facilitated by image thresholding. The list of marked loci can be labeled automatically, as can the readout of their Talairach coordinates and pixels values of anatomical and functional data sets. The BAFI also provides locus editing on the triplanar display, as well as importing and exporting of the locus list.

The BAFI is applicable for the following tasks: 1) rapid, atlas-based localization analysis of functional images; 2) correlation of structural and functional images (normalized to the Talairach space) enhanced by atlas-assisted labeling and triplanar display; and 3) interactive labeling of morphological images. The BAFI, it has been written, is also "useful in the clinical area for those involved in [fMR] image localization in surgical planning." 12

One key problem in localization analysis of functional images is the determination of Brodmann areas, gyri, and subcortical structures involved in the activation loci. A standard approach to this problem involves the use of the print version of the Talairach-Tournoux atlas, but this is tedious, error-prone, and time-consuming process. An electronic brain atlas equipped with suitable tools, such as those previously described, ${ }^{17,20,21}$ offers a solution to this problem. Even when an electronic atlas is used, however, identifying numerous activation loci on many functional images and reading their underlying cortical and subcortical anatomy, coordinates, and anatomical and functional values remains a tedious and time-consuming task.

We propose a novel, locus-driven mechanism by which to perform this task in an automated, rapid, explicit, consistent in $3 \mathrm{D}$, and user-friendly way. In this paper we describe the use of the BAFI for localization analysis of functional images, with a special emphasis on locus processing and locus-driven generation of results.

\section{MATERIALS AND METHODS}

\section{Generation and Analysis of Functional Images}

The overall process involved in the generation and analysis of functional images comprises four stages: 1) experimental design/patient study and functional image generation/acquisition; 2) volume reformatting to provide an axial, coronal, or sagittal orientation, if not available originally; 3 ) file format conversion, if necessary; and 4) atlas-assisted analysis (Fig. 1). There are a number of packages supporting the first three stages. ${ }^{21}$ Thus, our work focuses on the fourth stage.

\section{Atlas-Assisted Analysis of Functional Images}

One purpose of BAFI is to assist analysis of functional images in research and clinical applications. ${ }^{21}$ It enables the loading of anatomical and functional data, their correlation, identification of activation loci, and the labeling of these loci with Brodmann areas, gyri, and subcortical structures by means of an extended and enhanced version of the Talairach-Tournoux brain atlas. The Talairach proportional grid system transformation is used to normalize the anatomical and functional data and register them with the atlas. The availability of numerous tools supports this process (Fig. 2).

\section{Process of Data Analysis}

The BAFI supports processing of anatomical and functional data. The specification of "anatomical" image data requires only that this data set enables the identification of the necessary landmarks for definition of the Talairach proportional grid system transformation. Similarly, the "functional" data set can be any class of data that is in registration with the anatomical data set and can include statistical maps from functional imaging (fMR imaging and positron emission tomography scanning), metabolic maps (positron emission tomography and single-photon emission computerized tomography scanning), other anatomical or research maps, and maps of other parameters such as diffusion and perfusion.

The operations supporting an atlas-assisted analysis of anatomical and functional data are arranged in five processing steps: 1) Load data to select, verify, and load an anatomical data set; 2) Set Talairach landmarks for setting the landmarks on the anatomical images in 3D space; 3) Warp data to atlas to register the anatomical data set with the Talairach atlas by using the Talairach proportional grid system transformation (the functional data set can then be selected, verified, and loaded, and the same normalization transformation is applied to it); 4) Identify/edit activation loci (the activation loci are identified within the activation regions and their Talairach coordinates are read); and 5) Get labels/values (the activation loci are labeled with Brodmann areas, gyri, and subcortical structures, and additionally, the pixel values of the activation loci are read.

\section{Talairach Proportional Grid System}

The Talairach landmarks are set on the anatomical images, which subsequently are normalized by means of the Talairach proportional grid system transformation. The functional images, in which a position is known with respect to the anatomical images, undergo the identical transformation. The anatomical and functional images as well as the atlas plates are placed in the Talairach space, and they are displayed together as a single, blended data set. Table 1 provides a summary of abbreviations for the Talairach landmarks.

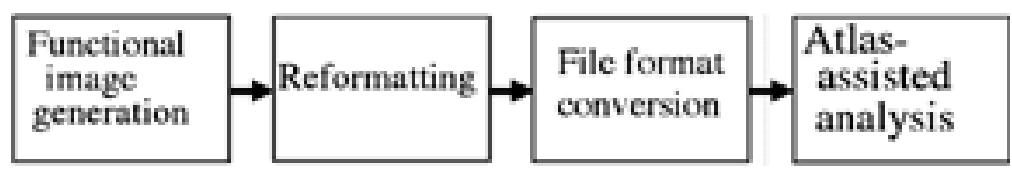

Fig. 1. Flow chart showing the process of generation and analysis of functional images. 


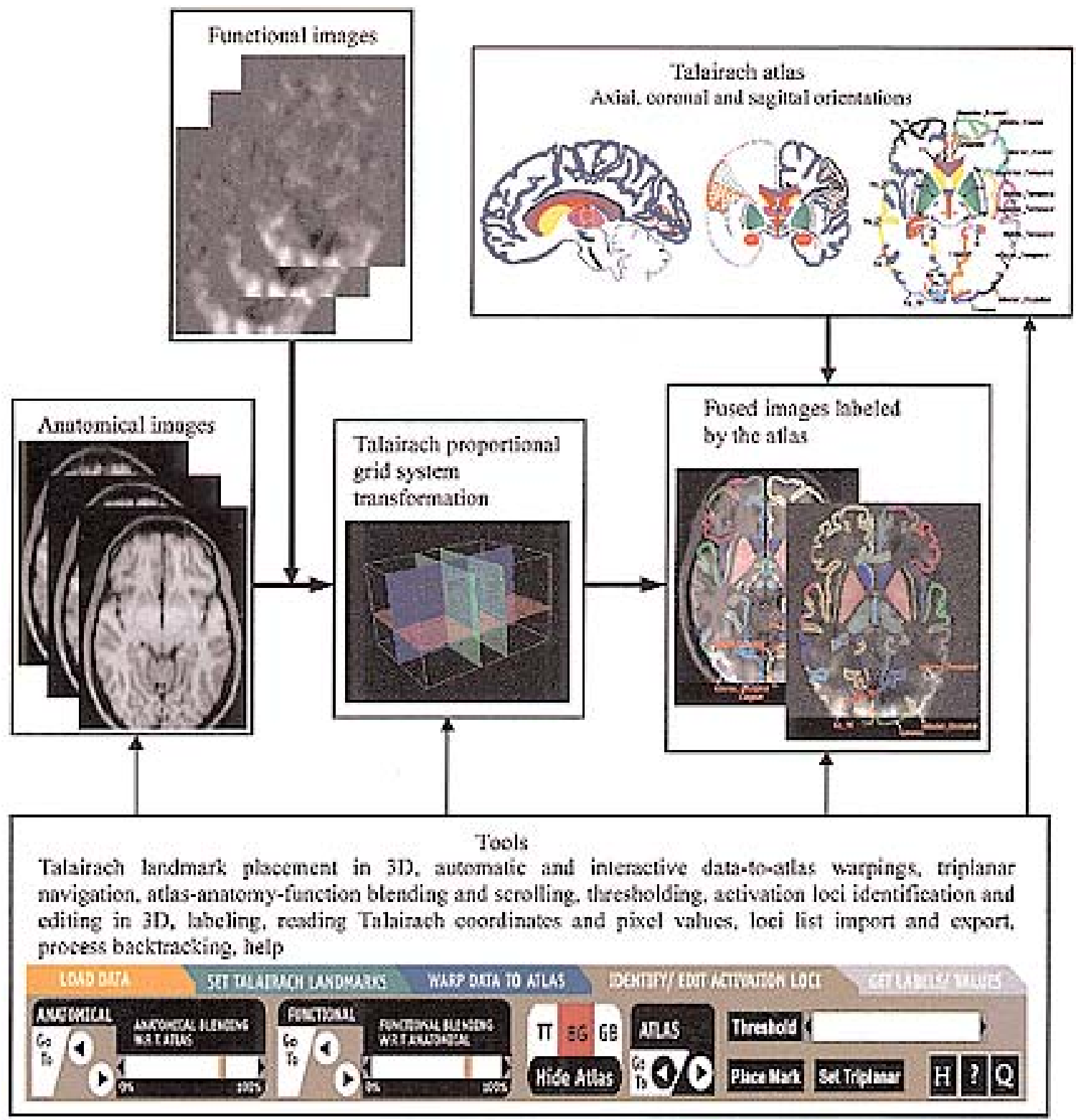

Fig. 2. Diagram illustrating data and control flow in the BAFI.

The landmarks divide the brain into 12 regions (Figs. 2 and 3). Within each region the brain images are warped piecewise linearly.

For the manually identified landmarks, the anatomical and functional images are warped automatically. Moreover, the process of interactive warping provides an additional deformation mechanism for fine tuning the fit of the data to the atlas. By dragging any Talairach landmark (rigidly attached to the data after the automatic transformation), the anatomical and functional images are being deformed in real time. In this way, the images may be better fitted into the atlas, either globally or locally.

\section{Electronic Talairach-Tournoux Brain Atlas}

To construct an electronic version of the Talairach-
Tournoux atlas, the original print plates were digitized with high resolution and extensively processed, enhanced, and extended, such that the following was conducted. 1) The original grids, rulers, and annotations were removed. 2) Each atlas structure was assigned a unique color-coded representation, as opposed to a mixture of contour, colorcoded, and texture representations in the print atlas. 3) The left thalamic subnuclei, not available in the print atlas in the axial and coronal plates, were outlined and color coded. 4) The right hemisphere cortex for axial orientation was added by mirroring the left hemisphere cortex. 5) Brodmann areas and gyri, which are labeled but not segmented in the print atlas, were constructed and color coded for axial orientation. The construction of the electronic atlas has been described in more detail in our previous papers. . $^{13,14,18}$ 
TABLE 1

Talairach landmarks used in the Talairach transformation

\begin{tabular}{cl}
\hline \hline Abbreviation & \multicolumn{1}{c}{ Landmark } \\
\hline AC & $\begin{array}{l}\text { anterior commissure } \\
\text { posterior commissure } \\
\text { PC }\end{array}$ \\
L & $\begin{array}{l}\text { most lateral point of the parietotemporal cortex for the } \\
\text { most lateral point of the parietotemporal cortex for the } \\
\text { R }\end{array}$ \\
At hemisphere \\
P & $\begin{array}{l}\text { most anterior point of the frontal cortex } \\
\text { most posterior point of the occipital cortex } \\
\text { h }\end{array}$ \\
highest, most superior (most dorsal) point of the \\
parietal cortex \\
lowest, most inferior (most ventral) point of the \\
temporal cortex
\end{tabular}

\section{Loci Processing}

Within each activation region of functional images, a "mark" is placed to determine the "activation locus" (focus). A mark represents its corresponding activation locus and it contains a "cross" which denotes the location of the locus, and a "consecutive number" identifying the locus. All activation loci form the "locus list." The activation locus is can be labeled if its mark lies within a color-coded region of the atlas. To meet this condition, the locations of marks can be edited. The mark to be edited is selected from the locus list and its editing can be undertaken on any orthogonal plane. Because locus editing is a 3D operation, it requires triplanar navigation in axial, coronal, and sagittal planes.

Three operations facilitate identification of the activation loci and their editing. 1) Threshold is for the thresholding of functional images containing the activation regions, which facilitates definition of the activation locus within the activation region. 2) Place Mark is for placing marks on images. Typically, a mark is placed on the activation locus within the activation region, and it identifies this locus only; in general, the user can place marks on any points of interest. 3) Set Triplanar is for controlling the triplanar and providing cross-referencing among orthogonal views. The triplanar display comprises the main view (where the operations are performed) and two smaller reference views. Because the main view can be swapped with any reference view, easy triplanar navigation is provided. The triplanar display can also be set from the locus list, such that its origin corresponds to the coordinates of the selected locus.

Each entry in the locus list corresponds to the activation locus identified by the user, and it contains the mark number (identifier), its Talairach (x, y, and z) coordinates, label (for subcortical structures) or labels (for cortical areas), and pixel values of the anatomical and functional data sets. Four functions are provided to operate on the locus list. 1) Edit Locus enables editing position of a selected locus by means of the arrow keys. 2) Delete Locus removes the selected locus from the locus list. 3) Clear List deletes the entire locus list. 4) Save List saves the locus list in an external file.

The user can control a rapid generation and display of results directly from the locus list. By clicking any entry
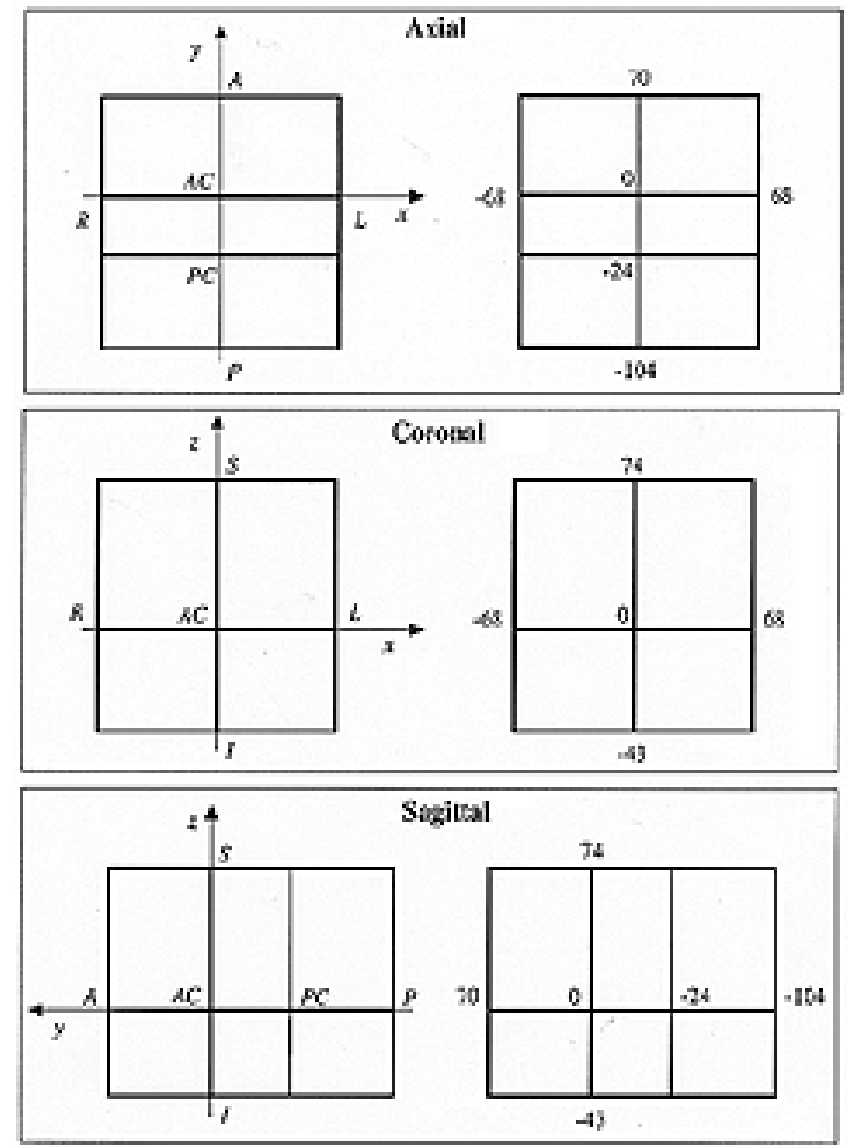

Fig. 3. The Talairach coordinate system, landmarks, and grid (in millimeters) on axial, coronal, and sagittal planes.

in the list, the following actions occur automatically. 1) The triplanar display is set to the selected locus. 2) The entry is updated with the labels and the pixel values of the anatomical and functional images (if the label does not exist, the message No Label appears in the entry, indicating that the position of this mark has to be edited). 3) The locus is labeled in the main view. 4) The locus pixel values of the anatomical and functional images are displayed below the main view.

\section{RESULTS}

The process of placing marks facilitated by thresholding is illustrated in Fig. 4. By decreasing the threshold from its maximum value, the most intensive location (corresponding to the most active locus) in the most active region becomes thresholded first (and is set to black). A mark is placed on it just by clicking it (Fig. 4 left). By keeping the threshold decreased, the subsequent activation regions get thresholded and their most active locations marked (Fig. 4 right).

The numbers of the subsequent marks are placed on the image (Fig. 4) and stored in the locus list with their Talairach coordinates (Fig. 5). After placing all marks on all images, these marks may additionally be edited by removing redundant marks (to avoid placing of multiple 


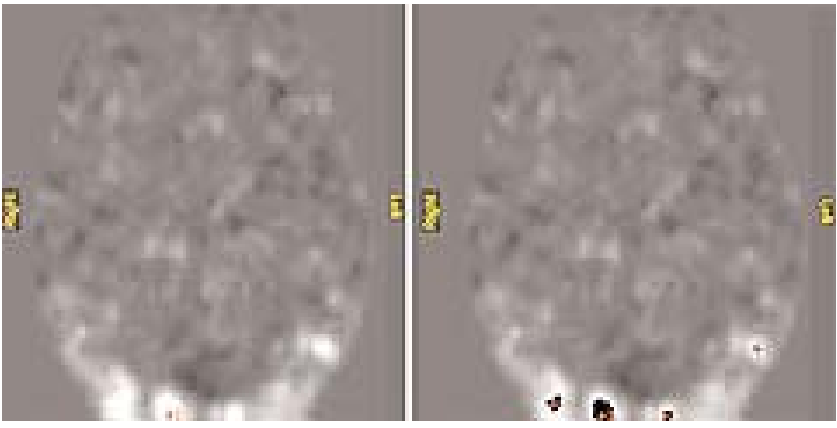

Fig. 4. Images demonstrating the placement of marks on subsequent activation loci of functional images by continuously decreasing the threshold;. Left: The first thresholded activation region with the mark placed. Right: The marks placed on subsequent activation regions.

marks within the same activation region, which may occur during processing of subsequent images).

When executing the last step (Get labels/values) of the image analysis process, any mark clicked in the locus list becomes labeled with the anatomical name(s), and the pixel values of the anatomical and functional data sets are read; the label(s) and values are stored in the loci list. Additionally, any mark that is clicked gets highlighted and labeled in the main view. Other structures can also be labeled by clicking their names in the anatomical index (Fig. 5).

\section{DISCUSSION}

Localization of numerous activation loci on many functional images and determining their underlying cortical and subcortical anatomy, coordinates, and anatomical and functional values is a tedious, time-consuming, and error-prone task. The aforedescribed locus-driven mechanism enables this task to be undertaken in an automated, rapid, explicit, three dimensionally consistent, and user-friendly way.

Several features make the locus-driven mechanism automated and user friendly. A mark can be localized on any orientation, and thresholding facilitates this operation. An interactive change of thresholding provides a simple way of finding the most active location within the activation region and allows the mark to be placed there. The mark is checked automatically if it is located on the atlas

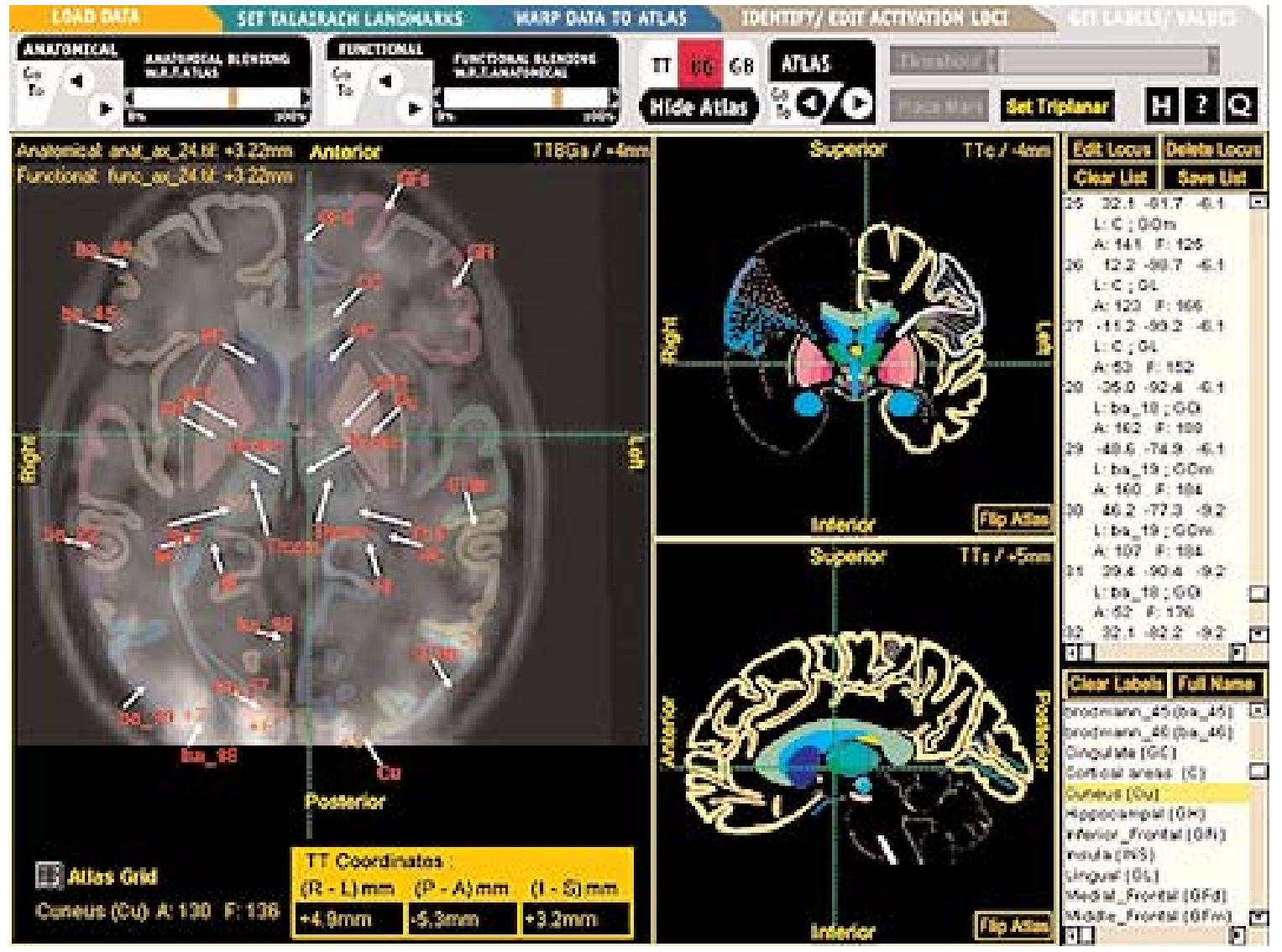

Fig. 5. User interface of the BAFI. Of the three images, the main view is shown at left and the two reference views at right. At the far right side of the Figure, the locus list and anatomical index are shown in the scrolling column. The control panel can be seen at the top of the screen. The anatomical, functional, and atlas images are blended and displayed together in the main view. The main view images are labeled with subcortical structures, gyri, and Brodmann areas. In addition, the triplanar display is set and the cross-reference lines displayed in all three views. 
to ensure that there is a label corresponding to this location. This operation can be conducted on the triplanar display easily, because clicking any locus on the locus list causes the origin of the triplanar to be set at the location of the mark. If the locus cannot be labeled, its position can be edited so that it is assigned a label (or labels). Editing of locus position is a $3 \mathrm{D}$ operation and can be performed in any orientation. The key advantage of the proposed mechanism is that the labels, Talairach coordinates, and pixel values for all loci are generated automatically and can be saved on an external file.

The BAFI displays the activation regions as well as the atlas. This explicit availability of the atlas overlaid on the data ensures that any activation locus is labeled. Note that when locus localization and its labeling are separated, as for instance in the BrainMap system, ${ }^{4}$ the information about the original activation region is lost, and no unique solution to the labeling problem is provided when the locus is placed initially outside the segmented atlas. Some regularization is then necessary to label the loci located outside the atlas structures. BrainMap, for instance, employs the Talairach Daemon ${ }^{11}$ that searches for the cortical area closest to the activation locus, and its label is assigned to the considered locus. The BAFI does not make any assumptions that may be invalid in a particular case and the user has full control over the selection of the activation locus within the activation region with respect to the explicitly displayed atlas.

The axial, coronal, and sagittal plates of the TalairachTournoux brain atlas are not consistent in the 3D space, as has been illustrated. ${ }^{19}$ Hence, the simultaneous use of all three atlas orientations to label any activation locus in 3D space may result in three different labels. To make labeling consistent and repeatable across experiments, individuals, and centers, we recommend using a single (reference) atlas orientation only. In the BAFI, the axial orientation has been chosen as the reference orientation and the original axial plates have been suitably extended. They contain the cortical areas for both hemispheres, fully color coded and labeled with Brodmann areas and gyri. Suitable tools have been provided to ensure 3D consistency including triplanar navigation, which facilitates crossreferencing among all three atlas orientations and locus editing in 3D space on any orthogonal plane.

The limitations of the Talairach-Tournoux atlas (including atlas plates, grid, and landmarks), Talairach transformation, and Brodmann areas are well known. We have discussed them in more detail in previous work. ${ }^{16,19}$ With a low degree of freedom transformation, the Talairach approach is not able to cope with a substantial variability of sulcal patterns, whereas its accuracy within the subcortical region is comparable to that obtained by nonlinear warping methods. For the given Talairach landmarks, because this transformation normalizes a neuroimage within a fraction of a second, processing of hundreds of anatomical and functional neuroimages can be accomplished quickly as opposed to requiring days when nonlinear methods are used. ${ }^{19}$

The placement of the Talairach landmarks in the BAFI is interactive. By combining our two patent-pending algorithms for a rapid and automatic detection of the midsagittal plane (Hu QM, Nowinski WL: patent application no. PCT/SG02/00006) and identification of the AC and PC landmarks on it (Nowinski WL, Thirunaruukarasuu A: patent application no. PCT/SG00/00185) with the modified Talairach landmarks, ${ }^{15}$ we hope soon to provide an automatic Talairach transformation that occurs within seconds.

Some other limitations of the BAFI include static labeling (meaning that the labels are placed in the predefined locations), lack of image zoom, and atlas availability in bitmap representation that obscures the original data when superimposed on it. Our recently described Cerefy Clinical Brain Atlas ${ }^{20}$ overcomes these atlas limitations.

\section{References}

1. Brodmann K: Vergleichende lokalisationslehre der grosshirnrinde in ihren prinzipien dargestellt auf grund des zellenbaues, Barth, Leipzig, 1909, in von Bonin G (ed): Some Papers on the Cerebral Cortex. Springfield, IL: Charles C Thomas, 1960, pp 201-230

2. Cox RW: AFNI: software for analysis and visualisation of functional magnetic resonance neuroimages. Comp Biomed Res 29:162-173, 1996

3. Eddy WF, Fitzgerald M, Genovese C, et al: Functional image analysis software, in Prat A (ed): Proceedings in Computational Statistics, (COMPSTAT 1996). Heidelberg: PhysicaVerlag, 1996, pp: 39-49

4. Fox PT, Mikiten S, Davis G, et al: BrainMap: a database of human functional brain mapping, in Thatcher RW, Hallett M, Zeffiro T, et al (eds): Functional Neuroimaging: Technical Foundations. San Diego: Academic Press, 1994, pp 95-106

5. Fox PT, Parsons LM, Lancaster JL: Beyond the single study: function/location metanalysis in cognitive neuroimaging. Curr Opin Neurobiol 8:178-187, 1998

6. Friston KJ, Holmes AP, Worsley KJ, et al: Statistical parametric maps in functional imaging: a general linear approach. Hum Brain Mapp 2:189-210, 1995

7. GE Medical Systems: FunctTool Operator Manual. General Electric Company, 1997

8. Goebel R: Brainvoyager: a program for analyzing and visualizing functional and structural magnetic resonance data sets. Neuroimage 3:S604, 1996 (Abstract)

9. Gold S, Christian B, Arndt S, et al: Functional MRI statistical software packages: a comparative analysis. Hum Brain Mapp 6:73-84, 1998

10. Lancaster JL, Fox PT: Talairach space as a tool for intersubject standarization in the brain, in Bankman I (ed): Handbook of Medical Imaging. Processing and Analysis. San Diego: Academic Press, 2000, pp 555-567

11. Lancaster JL, Woldorff MG, Parsons LM, et al: Automated Talairach atlas labels for functional brain mapping. Hum Brain Mapp 10:120-131, 2000

12. Lozano A: Book review of Nowinski WL, Thirunavuukarasuu A, Kennedy DN: Brain Atlas for Functional Imaging: Clinical and Research Applications. Singapore: Thieme, 2001. J Neurosurg 95:1105, 2001

13. Nowinski WL: Computerized brain atlases for surgery of movement disorders. Semin Neurosurg 12:183-194, 2001

14. Nowinski WL: Electronic brain atlases: features and applications, in Caramella D, Bartolozzi C (eds): 3D Image Processing: Techniques and Clinical Applications. New York: Springer-Verlag, 2002, pp 79-93

15. Nowinski WL: Modified Talairach landmarks. Acta Neurochir 143:1045-1057, 2001

16. Nowinski WL, Below D: The Cerefy Neuroradiology Atlas: a Talairach-Tournoux atlas-based tool for analysis of neuroimages available over the Internet. Neuroimage, 2003 (In press) 


\section{Atlas-assisted analysis of functional images}

17. Nowinski WL, Bryan RN, Raghavan R: The Electronic Clinical Brain Atlas. Multi-planar Navigation of the Human Brain. New York: Thieme, 1998

18. Nowinski WL, Fang A, Nguyen BT, et al: Multiple brain atlas database and atlas-based neuroimaging system. Comp Aid Surg 2:42-66, 1997

19. Nowinski WL, Thirunavuukarasuu A: Atlas-assisted localization analysis of functional images. Med Image Anal 5: 207-220, 2001

20. Nowinski WL, Thirunavuukarasuu A, Benabid AL: The Cerefy Clinical Brain Atlas on CD-ROM, ed 2. New York: Thieme, 2003

21. Nowinski WL, Thirunavuukarasuu A, Kennedy DN: Brain Atlas for Functional Imaging. Clinical and Research Applications. New York: Thieme, 2001

22. Sensor Systems: MEDx 2.1. Multimodality Radiological Im- age Processing for Unix Workstations. Sensor Systems Inc., 1998

23. Strupp JP: Stimulate: a GUI based fMRI analysis software package. Neuroimage 6:607, 1996 (Abstract)

24. Talairach J, Tournoux P: Co-Planar Stereotaxic Atlas of the Human Brain. New York: Thieme, 1988

Manuscript received May 15, 2003.

Accepted in final form June 13, 2003.

This work was funded by the Biomedical Research Council, Agency for Science, Technology and Research, Singapore.

Address reprint requests to: Wieslaw L. Nowinski, D.Sc., Ph.D., Biomedical Imaging Lab, Institute for Infocomm Research, 21 Heng Mui King Terrace, Singapore 119613. email:wieslaw@i2r.astar.edu.sg. 